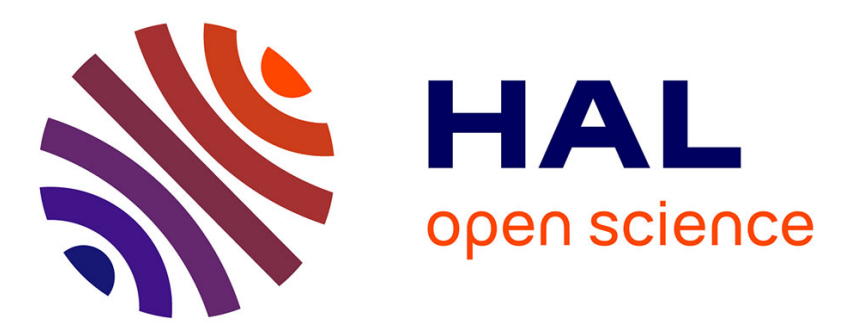

\title{
Vers l'épuisement d'un modèle de croissance Hong Kong et la crise asiatique
}

\author{
Jean Jaulin, Jean-François Huchet
}

\section{To cite this version:}

Jean Jaulin, Jean-François Huchet. Vers l'épuisement d'un modèle de croissance Hong Kong et la crise asiatique. Perspectives chinoises, 1998, pp.18 - 29. 10.3406/perch.1998.2292 . hal-02543962

\section{HAL Id: hal-02543962 \\ https://hal.science/hal-02543962}

Submitted on 15 Apr 2020

HAL is a multi-disciplinary open access archive for the deposit and dissemination of scientific research documents, whether they are published or not. The documents may come from teaching and research institutions in France or abroad, or from public or private research centers.
L'archive ouverte pluridisciplinaire HAL, est destinée au dépôt et à la diffusion de documents scientifiques de niveau recherche, publiés ou non, émanant des établissements d'enseignement et de recherche français ou étrangers, des laboratoires publics ou privés. 


\section{Vers l'épuisement d'un modèle de croissance [Hong Kong et la crise} asiatique ]

Hong Kong et la crise asiatique

Jean Jaulin, Jean-François Huchet

\section{Citer ce document / Cite this document :}

Jaulin Jean, Huchet Jean-François. Vers l'épuisement d'un modèle de croissance [Hong Kong et la crise asiatique ]. In: Perspectives chinoises, $n^{\circ} 47$, 1998. pp. 18-29;

doi : https://doi.org/10.3406/perch.1998.2292

https://www.persee.fr/doc/perch_1021-9013_1998_num_47_1_2292

Fichier pdf généré le 24/04/2018 


\title{
Hong Kong et la crise asiatique \\ Vers l'épuisement d'un modèle de croissance
}

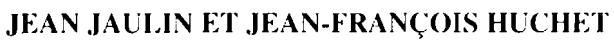

$\mathrm{R}$ APPELONS-NOL's : il y a un an. l'économic hongkongaise flamboyait : rien ne semblait pouvoir arrêter la montée des prix de l'immobilier et des cours de la bourse. le gouvernement annonçait des surplus budgétaires à faire pâlir plus d'un gouvernement occidental, les firmes chinoises se ruaient à la porte de la bourse pour offrir leurs titres qui, à chaque fois, étaient souscrits plusieurs centaines de fois par rapport au montant des actions disponibles : bref, au moment de la rétrocession, l'économie hongkongaise arborait fièrement ses « fondamentaux » économiques comme un premier de la classe, face à une mère patrie chinoise, certes en pleine croissance, mais au prise avec d'innombrables défis de modernisation, et qui avait tout à apprendre de l'économie du territoire.

Le contraste aujourd'hui. n'en est que plus frappant. Bien sûr, au moment de la rétrocession. on trouvait toujours quelques "mauvaises langues » pour prédire un retournement de conjoncture, mais peu auraient parié sur un mouvement aussi rapide et profond. Après avoir tenu dur comme fer jusqu'au mois de mai à une prévision de croissance de 3,5\% pour l'année 1998, alors que tous les observateurs parlaient déjà de récession. le gouvernement de la Région administrative spéciale (RAS) reconnaît enfin ouvertement que cet objectif ne sera pas atteint. Et, tout indique que l'économie hongkongaise s'enfonce dans une récession sévère et peut-être durable. L'indice Hang Seng de la bourse de Hong Kong a perdu près de $50 \%$ de sa valeur par rapport à l'été dernier (de 16000 à 8000 points à la mi-juin), entraînant derrière lui la fermeture de trois sociétés de courtage prises dans des scandales de corruption financière et la mise en liquidation de la plus grande société d’investissement de Hong Kong, Peregrine, dont une partie a été depuis rachetée par la BNP. L'immobilier et le tourisme ont chuté de 30 à $40 \%$. tandis que les ventes de détail se contractent de $20 \%$ et que la production industrielle est en baisse de plus de $10 \%$. Les firmes chinoises installées à Hong Kong ont vu la valeur de leurs actions s"effondrer ; les fameuses "Red Chips » (filiales d'entreprises chinoises installées à Hong Kong) ont pratiquement bloqué tous leurs projets d'expansion et de levée de capitaux : quant aux entreprises d'Etat chinoises (les « $\mathrm{H}$ » shares), leurs nouvelles émissions se font au compte-gouttes sur la bourse de Hong Kong après avoir représenté près des deux tiers des émissions dans la deuxième moitié de 1997, suite à une vague spéculative qui avait vu leurs cours doubler en un an. La crise financière s"est propagée rapidement dans la sphère productive avec une montée inquiétante du chômage qui. d'après les analystes de la place. serait largement sous-évaluée dans les chiffres officiels $(4,2 \%$ en mai dernier). Alors qu au moment de la rétrocession, c'était Hong Kong qui devait aider la Chine. les autorités de la RAS cherchent maintenant à attirer les touristes de Chine afin de compenser la chute du tourisme asiatique et même européen. Elles scrutent surtout avec attention les tendances de l'économie chinoise. seule capable à leurs yeux d'enrayer. aujourd hui, la récession. Une façon, pour certains analystes, d'éviter de s'attaquer à quelques déséquilibres importants nés d'un boom spéculatif majeur qui a débuté en 1995 et s'est nettement amplifié à partir de janvier 1997. et plus fondamentalement sans doute, d'avoir à résoudre l'équation du Territoire dans la décennie à venir : comment continuer à bénéficier de la rente d'entrée sur un marché de 1,2 milliard de personnes après lui avoir servi de pilote d'expérimentation dans les réformes de marché, et notamment sur le plan financier.

Que s'est-il donc passé depuis un an? Quels sont les facteurs qui expliquent la crise actuelle? S'agit-il d'un simple retournement de conjoncture ou d'une crise plus profonde susceptible de modifier les mécanismes de régulation de l'économie hongkongaise ainsi que le comportement des acteurs ? Quelles interactions avec la nouvelle donne politique, issue de la rétrocession et des élections législatives du 24 mai dernier, peut-on anticiper à moyen terme ? Nous tenterons de répondre à ces questions en essiayant de démontrer que si la crise asiatique a joué un rôle important dans le déroulement de celle de Hong Kong. elle a également révélé au grand jour et exacerbé certains vices fondamentaux dans le mécanisme de la croissance. En effet. Hong Kong a longtemps été présentée comme une simple victime de la crise asiatique. Cependant. une analyse plus précise de la situation 


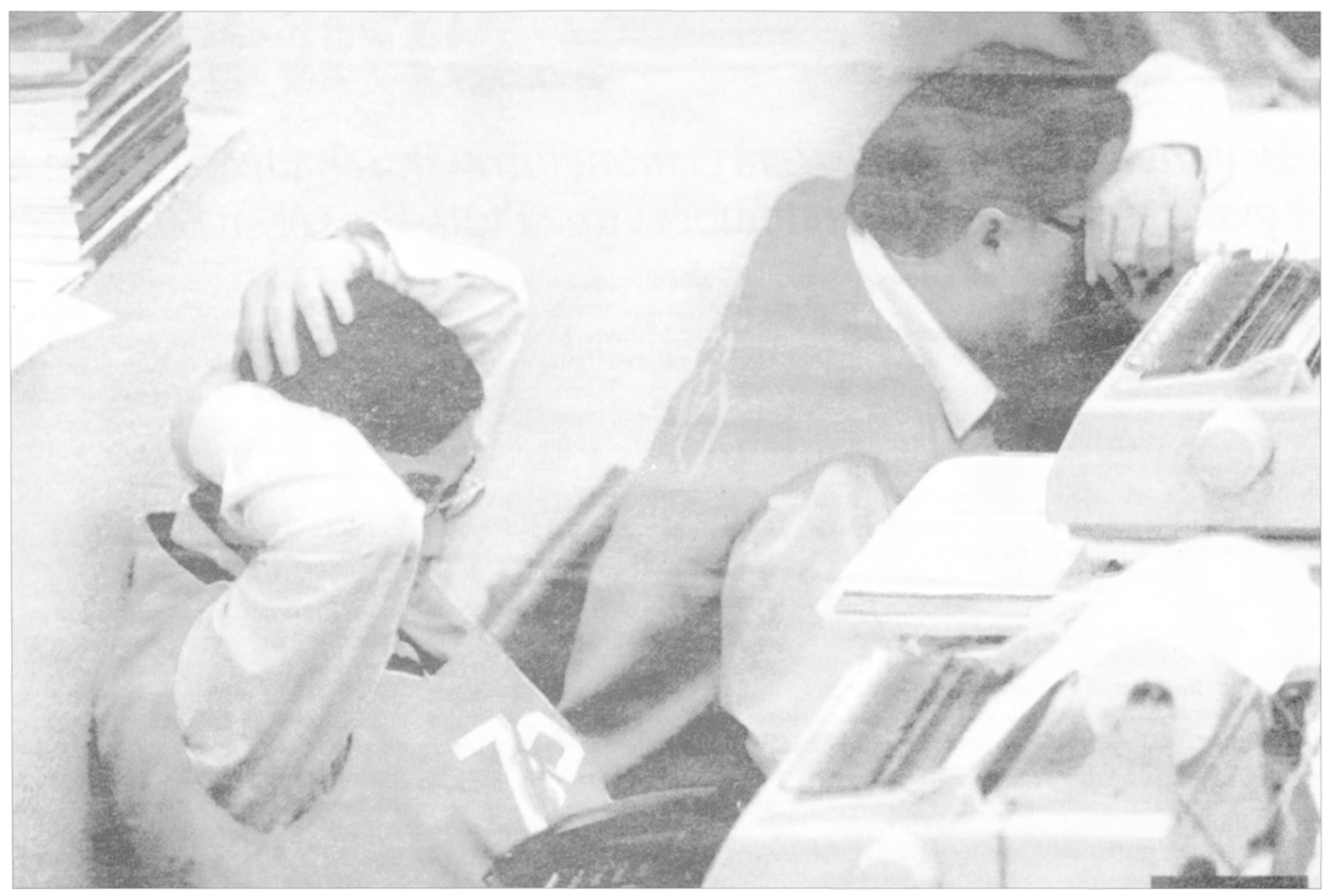

Un an après la rétrocession, la fête est bien finie à la bourse de Hong Kong

montre que la crise en Asie a accéléré l’éclatement d’une bulle spéculative dans l'immobilier et aiguisé les défis liés à la rétrocession, soit un ensemble de problèmes qui étaient déjà perceptibles à Hong Kong au moment de la dévaluation de la monnaie thaïlandaise le 2 juillet 1997. De ce fait, et contrairement à ceux qui pensent qu'un retour à une croissance soutenue se fera de manière rapide et indolore par les simples jeux de la flexibilité des marchés, on peut sans doute montrer au contraire que des issues saines et durables ne pourront vraisemblablement être trouvées sans des ajustements profonds. dont certains dépassent largement la sphère économique.

\section{Les conséquences directes de la crise asiatique}

\section{La contagion financière}

Hong Kong ne pouvait tout à la fois revendiquer le statut de deuxième place financière en Asie et considérer qu'elle ne serait pas affectée par la crise asiatique comme elle $l^{\circ}$ a fait pendant plusieurs mois. Sur un plan purement interne. le système financier hongkongais fait. en outre. partie intégrante de la crise asiatique en raison d'un mode de croissance depuis 10 ans qui repose entièrement sur l'immobilier. la bourse et leurs collatéraux. Ces derniers ont permis l'obtention de crédits bancaires en croissince rapide et ayant comme ailleurs bénéficié de taux d’intérêts réels négatifs pendant plusieurs années en raison du différentiel d'inflation positif avec les Etats-Unis qui vient de se retourner brutalement en quelques mois.

La place financière de Hong Kong remplit essentiellement trois fonctions :

- un centre offshore. le ler du monde, avec 212 milliards de SUS d'engagements des banques occidentales déclarantes à la Banque des règlements internationaux (BRI), auxquels s'ajoutent les engagements des autres pays non déclarants, qu'il sagisse d’autres places offshore comme les Iles Caïmans ou Singapour (35,4 milliards fin 1997). ou d'autres pays asiatiques comme la Corée ( 8 milliards). la Thaïlande et même l'Indonésie. Au total, les engagements des banques étrangères représentaient, en mars 1997. 66.6\% des dépôts totaux à Hong Kong, contre $2.2 \%$ en Chine. $44 \%$ en Corée. et $262 \%$, le record. à Singapour. en raison de son rôle privilégié de marché des changes régional plus que de centre de fïnancement bancaire comme à Hong Kong.

- un centre de transformation bancaire pour la Chine avec des engagements sur celle-ci qui s'élèvent à 33.8 milliards de SUS en devises et à 17 milliards en équivalent SHK. et son rôle privilégié pour les investissements directs étrangers (IDE) vers la Chine $(57 \%$ des 45 milliards en 1997) comme en provenance de Chine (10 milliards de 1985 à 1995 selon la Cnuced - 1997).

- un centre financier autochtone de premier plan dans la région avec une capitalisation boursière locale de 595 
milliards de \$US au pic de 1997, soit $341 \%$ du PIB, et donc le plus élevé du monde (18\% en Chine, $211 \%$ à Singapour), et une masse de crédits locaux évalués à 288 milliards de \$US en décembre dernier, soit $169 \%$ du PIB, là encore les plus élevés d'Asie à égalité avec la Thaillande.

La crise asiatique a provoqué une cassure dans ces trois domaines :

1. On a assisté à une contraction globale des financements internationaux sur l'Asie (crise de liquidité) et à une montée vertigineuse des risques (crise de solvabilité). Du côté des banques déclarantes à la BRI, les retraits de capitaux de la place offshore ont atteint, au cours du deuxième semestre 1997, 35,8 milliards \$US dont presque un tiers ( 10,3 milliards) pour la place de Hong Kong. On estime à 24,3 milliards les retraits totaux à Hong Kong, y compris ceux des pays non déclarants. Si les retraits japonais ont bien été prédominants $(-11$ milliards), les banques allemandes n'ont pas été en reste (4,2 milliards), ni celles d'Italie $(-1,3$ milliard $)$, d'Espagne $(-1,2)$, etc. Seules les banques françaises ont en fait augmenté leurs engagements ( $+4,2$ milliards).

2. Les effets de la crise sur la diaspora chinoise en Asie et la montée des inquiétudes sur la capacité de la Chine à résister à la déflation régionale se traduisent par des craquements comme ceux de Peregrine ou du groupe sino-thaï C.P. Pokphand (coté à Hong Kong). Celui-ci a été pressé par ses créanciers de se délester de ses acquisitions en Chine pour faire face à des obligations bancaires supérieures à 1 milliard de \$US. Cela concerne également la viabilité des investissements réalisés en Chine, dans l'immobilier notamment, et ceux réalisés à Hong Kong, souvent au pic de la bourse et du marché immobilier. La chute du cours des sociétés chinoises cotées à Hong Kong, la rétrogradation de leur notation par les agences internationales comme Moody's, la pression des banques étrangères pour réduire leurs lignes de crédit sur des groupes qui apparaissent désormais surendettés au regard de leurs fonds propres (CITIC, China Everbright, etc.) participent de ce mouvement de credit crunch qui est également l'expression des doutes des investisseurs quant à la capacité des autorités locales à superviser la qualité des engagements sur la place. Or, la crise de la bourse, dont l'indice Hang Seng pourrait tomber à un plancher de résistance estimé à 6-7000 contre 16673 au sommet de 1997, et surtout le maintien de taux d'intérêts supérieurs à $10 \%$, ont cassé tout le ressort de la place financière pour ses opérations vers la Chine. Plus aucune émission substantielle n'a ainsi vu le jour depuis le krach du 23 octobre, les crédits syndiqués encore montés restent en fait «collés » dans les pools bancaires qui les ont montés, et les groupes chinois sont obligés d'aller sur le marché international (New York ou Londres) pour placer leurs émissions d'obligations convertibles, avec comme contrepartie, une exigence de transparence beaucoup plus grande qu’à Hong Kong dans les années passées.

3. Enfin, est apparue une crise spécifique du Hong Kong dollar et de son système financier interne. Son issue dépendra largement de la capacité de gestion des autorités de la R.A.S. sur les variables qui les concernent : ajustement ou non du prix des actifs. La Standard \& Chartered Bank vient ainsi d'estimer à 2 millions par ménage, la perte de richesse due au dégonflement des bulles immobilières et mobilières. La faillite de plusieurs maisons de courtage, dont C.A. Pacific pour la plus retentissante, n'a fait que rappeler l'importance de la pyramide de crédits qui étaient adossés à de l'immobilier ou à des portefeuilles boursiers (cf. infra). Dans ces conditions, le système financier de Hong Kong ne peut être que frappé par le même credit crunch et la montée du risque de solvabilité réduisant par là même sa capacité à financer la Chine et les autres pays de la région. C'est ce qui explique du reste le repli des établissements financiers internationaux qui réduisent sérieusement leurs effectifs après avoir attendu quelques mois pour évaluer la sévérité de la crise financière dans la région et à Hong Kong. En outre, déjà très perceptible pour les entreprises de taille moyenne, dont une grande part se sont délocalisées en Chine, mais aussi au Vietnam et ailleurs, le credit crunch commence à gagner les grands groupes hongkongais, y compris les plus importants comme Cheung Kong, New World, etc. Ces groupes constituaient l'une des principales justifications de la notion de la «porte d'entrée privilégiée sur la Chine ».

La principale variable qui cxprime aujourd'hui la pression sur le système financier hongkongais ce sont les taux d'intérêts. Ils seront en moyenne de $10 \%$ pour l'année, pour une inflation qui pourrait tomber à $2 \%$, soit un retournement brutal des taux réels au détriment des débiteurs qui voient dans le même temps s'effondrer la valeur de leurs actifs. Deux facteurs pourraient néanmoins éviter à la R.A.S. une crise financière majeure :

- une liquidité plus importante des grands groupes hongkongais, mais aussi des entreprises de taille moyenne, rarement trop endettées pour celles qui travaillent notamment à l'exportation ;

- une gestion plus saine des banques hongkongaises, échaudées elles aussi par les crises périodiques qui ont frappé le territoire depuis les années 50, et dont le taux de mauvaises créances devrait monter à $10-15 \%$ au pire, dont $50 \%$ de recouvrables. En comparaison d'un ratio de fonds propres de $14 \%$ environ, la probabilité d'une crise systémique du système bancaire reste très faible, en dépit de quelques faillites bancaires inéluctables. Mais l'ajustement pour le système financier qui devrait durer au mieux deux ans, et être plus sévère qu'en 1974 ou 1983 (cf. infra), va notablement réduire la surface régionale de Hong Kong dans les années à venir, la mettant en concurrence avec les plus grandes places mondiales, mais aussi avec Shanghai qui va bénéficier de la volonté des autorités chinoises de moderniser leur système 
financier et d'utiliser davantage leurs ressources d'épargne en renminbi. La publication en mars dernier d'une étude comparative sur les centres offshore en Asie par le Hong Kong Trade Development Council (HKTDC), comporte ainsi un tableau comparatif avec les réformes financières envisagées à Singapour qui montre le défi de cette place concurrente pour l'ensemble de l'Asie du sud-est. A cela s’ajoutent le retrait structurel des banques japonaises et une inquiétude des investisseurs taiwanais qui ont émigré ces dernières années vers d'autres paradis fiscaux comme les îles Vierges. La réduction de l'espace financier régional est donc un réel risque pour Hong Kong.

\section{La contagion commerciale}

Hong Kong est un nain commercial pour ses exportations (27,4 milliards de \$US en décembre 1997, soit l’équivalent de la Pologne, 23ème exportateur mondial), mais un géant mondial pour les échanges commerciaux qui transitent par ses installations portuaires et aéroportuaires (188,1 milliards, soit le 5ème rang mondial, devant Singapour). A ces flux commerciaux s'ajoutent les flux de services, notamment ceux du tourisme dont le nombre multiplie par 2,5 celui des consommateurs locaux.

La dépendance considérable vis-à-vis de l'Asie est loin de se limiter aux réexportations chinoises comme on le croit souvent. Sur 443,9 milliards \$HK de réexportations en 1997, la Chine continentale en représentait $35,7 \%$, les USA $20 \%$, et le reste de l'Asie près de $25 \%$. $\mathrm{Si}$ on prend le total des exportations, la part de l'Asie, Chine comprise, est de $76,7 \%$, soit la dépendance la plus élevée dans toute la région. Le choc de la crise dans ce domaine est à deux détentes:

- à court terme, la déflation asiatique et la contraction des échanges intra-asiatiques, dont Hong Kong était un "centre " essentiel, se traduisent par un manque à gagner important sur deux tableaux : la chute des importations asiatiques, notamment dans des pays comme la Corée $(-40 \%)$ se traduit par une diminution notable du trafic de réexportation régional, tandis que la faible poussée des exportations vers le reste du monde, en raison des difficultés liées à la crise financière de ces pays, ne compense pas cette diminution des flux intra-régionaux ; les exportations chinoises qui avaient connu un boom dans les années postérieures à la dévaluation de $1994(+25,8 \%$ encore en 1997) devraient. de l'avis de tous les experts, connaître un sérieux ralentissement autour de $5 \%$, voire moins pour les plus pessimistes (elles ont baissé de $1.5 \%$ en mai dernier). Compte tenu d'une détérioration des marges liée à une abondance de produits exportables, asse $z$ souvent similaires, la pression va être très forte sur les coûts de transport, tandis que de nombreux transporteurs locaux asiatiques ont des capacités libérées par la contraction des volumes exportés (tout particulièrement ceux de Corée). Or. Hong Kong figure très largement en tête des coûts unitaires de fret dans la région, de sorte qu'elle devrait subir un effet renforcé de ralentissement.

- à moyen terme, la concurrence régionale va se renforcer, et notamment vis-à-vis des ports chinois où les Hongkongais eux-mêmes ont souvent investi (notamment M. Li Ka-shing). Il s'agit tant des ports côtiers de toute la Chine, que des ports du sud, avec notamment ceux de Shenzhen dont le trafic double chaque année, alors que celui de Hong Kong est passé d'une croissance annuelle de $15 \%$ à $4 \%$ l'an dernier, et devrait être en recul cette année. Le phénomène était déjà marqué en 1997 avec une croissance importante du trafic de direct shipment qui ne s'enregistre plus comme réexportations de Hong Kong. Il en est de même pour le trafic avec Taiwan qui dispose désormais de l'ouverture d'une liaison directe entre Kaohsiung et la région de Xiamen. Une autre illustration est la multiplication des aéroports de classe internationale tant au sud (cinq désormais) que dans toute la Chine, au moment même de l'ouverture de l'immense aéroport de Chek Lap Kok.

\section{Le maintien de la parité coûte que coûte}

Lorsque le système du peg et du currency board (directoire monétaire) ont été mis en place en 1984, l'idée était que dans une économie de taille réduite comme celle de Hong Kong, l'établissement d'une monnaie stable encouragerait le développement des activités liées à la finance, tout en permettant également, en cas de chocs externes, des ajustements rapides par les prix et les salaires. Ce système a bien fonctionné, permettant à Hong Kong de devenir en l'espace de quinze ans, la deuxième place financière d'Asie et de surmonter sans trop de problèmes, les multiples chocs externes, notamment le crack boursier de New York en 1987, ou les crises de confiance liées aux négociations sur le statut de Hong Kong entre Britanniques et Chinois. Dans le cadre d'une parité fixe, dès que les investisseurs étrangers ou locaux expriment un sentiment de doute (fondé ou fictif) sur l'économie hongkongaise, les taux d'intérêts augmentent afin de calmer les attaques et les anticipations à la baisse sur le \$HK. Le système fonctionne assez bien dans le cas de crises de courte durée car les taux d'intérêts reviennent rapidement à leurs niveaux antérieurs. Mais il comporte également deux inconvénients.

D'une part, il ne permet pas de compenser par une dévaluation une augmentation rapide de prix dans certains secteurs conduisant à une dégradation de la compétitivité à moyen terme. La croissance vertigineuse des prix de l'immobilier à partir de la fin des années 80 (de $300 \%$ à $400 \%$ selon les quartiers entre 1987 et 1997 pour le prix de location des bureaux) a contribué à faire de Hong Kong, en l'espace de dix ans, la ville la plus chère du monde après Tokyo. Une dévaluation par les autorités britanniques avant la rétrocession. ou de la RAS juste avant le mois d'octobre 1997, aurait pu permettre de limiter la perte de compétitivité du territoire. 
Tableau 1 - Engagements des banques déclarantes à la BRI sur la place Offshore de Hong Kong

\begin{tabular}{|l|ccccc|}
\cline { 2 - 6 } \multicolumn{1}{c|}{} & $\begin{array}{c}\text { Engagements } \\
\text { internationaux } \\
\text { en Mds USD }\end{array}$ & $\begin{array}{c}\text { dont banques } \\
\text { japonaises } \\
\text { en Mds USD }\end{array}$ & $\begin{array}{c}\text { Poids dans } \\
\text { le total } \\
\%\end{array}$ & $\begin{array}{c}\text { Total des } \\
\text { engagements } \\
\text { à court terme } \\
\text { en Mds USD }\end{array}$ & $\begin{array}{c}\text { Poids dans } \\
\text { le total } \\
\%\end{array}$ \\
\hline déc. 95 & 240 & 133 & $55,4 \%$ & 208 & $86,7 \%$ \\
déc. 96 & 207 & 87,5 & $42,3 \%$ & 170 & $82,1 \%$ \\
déc. 97 & 212 & 76,3 & $36,0 \%$ & 168 & $79,2 \%$ \\
\hline
\end{tabular}

Source : BRI, mai 1998.

D'autre part, si un choc externe se prolonge comme c'est le cas depuis l'été 1997, la montée des taux d'intérêts a le temps d'avoir des conséquences sur la sphère réelle de l'économie et accentue le ralentissement de l'activité au moment où l'économie en aurait le plus besoin. Déjà, plusieurs mois avant la crise, les taux d’intérêts réels étaient supérieurs à ceux en vigueur aux Etats-Unis. Mais, depuis l'été 1997, ils se situent à des niveaux qui étouffent lentement l'économie du territoire, soit à trois ou quatre points supérieurs aux taux américains pour les taux à long terme, alors qu'en période normale la différence ne dépasse pas un quart de point. Les premières conséquences sont d'abord d'ordre financier avec une chute des cours de la bourse qui provoque dans son sillon une destruction de richesse et une baisse de la valeur des collatéraux qui servent dans la garantie des investissements. La situation financière des entreprises se dégrade également par le biais du renchérissement du crédit. Celle des ménages pâtit également de cette hausse des taux d'intérêts avec une augmentation sensible des coûts de remboursement des prêts immobiliers et une diminution de la valeur de leur hypothèque.

Cependant, à partir d'un certain stade, ces effets se combinent, s'alimentent les uns les autres, et finissent par créer des effets dévastateurs sur l'économie réelle : augmentation des faillites, montée du chômage (le taux officiel pourrait atteindre 6 à $7 \%$ à la fin de l'année), augmentation de l'épargne de précaution des ménages, et diminution de la consommation. Par ailleurs, le retrait des banques japonaises et européennes dans les opérations de crédits aux entreprises et de refinancement sur le marché bancaire de Hong Kong n'a rien arrangé. Dans un contexte où l'on risque de voir une augmentation rapide des défauts de paiements avec la chute de la bourse (et la destruction de richesse qui l'accompagne traditionnellement), et l'augmentation des montants de remboursement par les ménages sur les crédits liés à l'immobilier, les banques locales sont de plus en plus prudentes. Elles cherchent à diminuer leurs prêts et demandent (comme la loi à Hong Kong le leur autorise) le remboursement anticipé des prêts accordés aux entreprises afin de diminuer leur exposition au risque. Le résultat est que l'économie de Hong Kong est aujourd'hui enfermée dans un cercle vicieux et personne, dans les conditions actuelles de turbulences économiques en Asie, ne semblent à même de prédire quand il sera enrayé.

En faisant de la parité actuelle du Hong Kong dollar une question politique, les autorités de la RAS ont retiré elles-mêmes de leurs cartes un atout important, à savoir la possibilité d'utiliser une dévaluation comme outil de politique économique. Dans une interview à la $\mathrm{BBC}$ le 25 juin dernier, Sir Donald Tsang, le secrétaire aux Finances, déclarait une nouvelle fois qu'il démissionnerait si la parité venait à changer. La rétrocession avait en effet focalisé les attentions des autorités chinoises, mais aussi britanniques, sur la parité actuelle comme un des indicateurs les plus importants de la richesse de Hong Kong et de la supériorité de ses institutions. Ceci a contribué à associer la parité actuelle au destin du système du peg proprement dit et de ses institutions comme le currency board qui, jusqu'à preuve du contraire, pourraient parfaitement continuer à exister après un réajustement de la parité. Avant la rétrocession, ni les autorités britanniques ni les autorités chinoises n'ont voulu considérer la possibilité d'utiliser une dévaluation pour compenser les effets de la croissance vertigineuse des prix de l'immobilier. Les uns auraient accusé les autres de vouloir porter atteinte à la supériorité des institutions de Hong Kong.

Par ailleurs, depuis le début de la crise asiatique, les autorités de la RAS (avec l'assentiment ou sur les instructions de Pékin) ont fait le pari que le choc externe, provenant de la crise asiatique, serait de courte durée. «La crise sera terminée à Noël » disait-on à l'automne dernier dans l'entourage du pouvoir. Cela permettait en effet de faire jouer, sans trop de casse au niveau social, la flexibilité des marchés par le jeu de la hausse des taux d'intérêts et la baisse des salaires et des prix, en espérant que tout reviendrait dans l'ordre après quelques mois difficiles. Or, les autorités de la RAS, ont largement sous-estimé, à la fois la portée et la durée des chocs 
Tableau 2 • Evolution de la capitalisation boursière de Hong Kong de décembre 1996 à mai 1998

\begin{tabular}{|l|c|c|c|c|c||}
\hline & $\begin{array}{c}\text { Capitalisation } \\
\text { boursière } \\
\text { en Mds USD }\end{array}$ & $\begin{array}{c}\text { Poids dans } \\
\text { le PIB } \\
\%\end{array}$ & $\begin{array}{c}\text { Evolution } \\
\text { base } 100=\text { déc. 96 } \\
\text { indice }\end{array}$ & $\begin{array}{c}\text { Evolution par } \\
\text { rapport à déc. } 96 \\
\text { en Mds USD }\end{array}$ & $\begin{array}{c}\text { Poids dans } \\
\text { le PIB courant } \\
\%\end{array}$ \\
\hline $\begin{array}{l}\text { Décembre } 96 \\
\text { Juillet } 97\end{array}$ & 449 & $291 \%$ & $100 \%$ & 0 & $0 \%$ \\
Décembre 97 & 595 & $341 \%$ & $132 \%$ & 146 & $83 \%$ \\
Mai 98 & 413 & $236 \%$ & $92 \%$ & -36 & $-21 \%$ \\
\hline
\end{tabular}

Source : Hong Kong and Shanghai Banking Corporation, James Capital, juin 1998.

externes et leur combinaison explosive avec les problèmes internes de l'économie Hongkongaise.

En fait, à l'heure actuelle, les autorités de la RAS sont condamnées à tomber de Charybde en Scylla. Car si les autorités décidaient aujourd'hui de changer la parité, ou pire encore, de mettre fin aux institutions qui soutiennent le système du peg en pleine turbulence monétaire asiatique, cette décision pourrait avoir des conséquences difficilement prévisibles pour l'économie hongkongaise. Après avoir répété à chaque occasion possible que la parité serait maintenue coûte que coûte, une dévaluation enverrait sur le marché un signal très négatif. Etant donné la politisation de la parité, une dévaluation pourrait se traduire, pour les résidents de Hong Kong, en une perte de confiance dans la capacité du gouvernement à maintenir la valeur de leur monnaie. Bien qu'une crise généralisée de paiement de type coréen ou indonésien soit fort peu probable en raison des réserves en devises (qui couvrent presque deux fois la masse monétaire en $\$ \mathrm{HK}$ ) et du faible endettement des banques, les résidents pourraient transformer massivement leurs avoirs en \$HK en devises étrangères (principalement en \$US). Les autorités seraient alors vraisemblablement obligées d'utiliser une partie non négligeable des réserves et de maintenir les taux d'intérêts à un niveau élevé pour défendre le \$HK. Aussi, à ce stade de la crise et compte tenu de l'instabilité monétaire en Asie, les autorités de la RAS semblent condamnées à maintenir la parité actuelle. Il est évidemment difficile de refaire l'histoire et de savoir si une dévaluation avant la rétrocession, ou juste avant que la bourse ne s'effondre en octobre dernier, aurait diminué les effets de la crise asiatique et limité sa propagation dans l'économie du territoire comme l'expérience taiwanaise pourrait le laisser penser. Toujours est-il qu'en choisissant le maintien de la parité, les autorités de la RAS savaient délibérément qu'en cas de prolongement des chocs externes, elles exposaient Hong Kong à un ajustement drastique de la valeur des actifs boursiers, des prix et des salaires avec des conséquences économiques et sociales bien difficile à prévoir.

\section{Le dégonflement de la bulle immobilière}

Hong Kong est souvent dépeint à tort comme la ville où prédominent le commerce et la finance. Un découpage plus fin des nomenclatures statistiques nous permet d'observer une toute autre image. L'immobilier est en fait de loin le secteur d'activité le plus important dans l'économie hongkongaise. Si l'on ajoute les deux catégories liées à l’immobilier, figurant dans les recensements économiques annuels, à savoir celle nommée "activités immobilières » (développement, location, gestion de parcs immobiliers) qui représente $12,4 \%$ du PNB et l'activité (que l'on passe souvent sous silence) de location des personnes privées qui représente $12,7 \%$ du PNB, c'est donc un peu plus d'un quart du PNB qui était lié à l'activité immobilière en 1995. Le commerce, qui arrive en deuxième position, ne représentait que $17,1 \%$ du PNB et la finance $10,6 \%$ pour la même

\section{Tableau 3 - Prix de la location des bureaux dans le monde en 1996 ef 1997}

\begin{tabular}{|lcc|c|c|}
\hline & $\begin{array}{c}\text { décembre } \\
\mathbf{9 6}\end{array}$ & $\begin{array}{c}\text { décembre } \\
\mathbf{9 7}\end{array}$ & $\begin{array}{c}\text { Evolution } \\
\mathbf{9 7} / \mathbf{9 6}\end{array}$ & $\begin{array}{c}\text { Prix relatif } \\
\text { Tokyo=100 }\end{array}$ \\
\hline Tokyo & 1560 & 1307 & $-16 \%$ & $100 \%$ \\
\hline Hong Kong & 1240 & 1000 & $-19 \%$ & $77 \%$ \\
\hline Bombay & 1197 & 886 & $-26 \%$ & $68 \%$ \\
\hline Londres & 1076 & 1051 & $-2 \%$ & $80 \%$ \\
\hline Singapour & 748 & 871 & $16 \%$ & $67 \%$ \\
\hline Pékin & 697 & 499 & $-28 \%$ & $38 \%$ \\
\hline Sydney & 635 & 400 & $-37 \%$ & $31 \%$ \\
\hline Shanghiai & 624 & 383 & $-39 \%$ & $29 \%$ \\
\hline New York & 563 & 444 & $-21 \%$ & $34 \%$ \\
\hline Kunala Lumpur & 323 & 170 & $-47 \%$ & $13 \%$ \\
\hline Bangkok & 298 & 150 & $-50 \%$ & $11 \%$ \\
\hline
\end{tabular}

Source : Healey \& Baker, USS par $\mathrm{m}^{2}$ et par an. 
année. De manière encore plus significative, l’immobilier est, depuis le milieu des années 80 , l'activité qui contribue le plus à la croissance du PNB et des revenus : elle contribuait à hauteur de $22,2 \%$ entre 1985 et 1990 . puis de $31,5 \%$ entre 1990 et 1995 (avec des pics à $43 \%$ en 1994), loin devant le commerce avec $17,5 \%$ et $19,6 \%$ et la finance avec $7,5 \%$ et $15,7 \%$ pour les mêmes périodes". Ces quelques chiffres suffisent à montrer que l'immobilier est loin d'être un secteur d'activité parmi d'autres dans l'économie hongkongaise. Il constitue en fait le principal mécanisme sur lequel a reposé la croissance depuis le milieu des années 80. L'importance de l'immobilier sur la croissance des marchés financiers, des revenus fiscaux, des profits bancaires, des revenus des ménages est telle, que l'effondrement du marché, auquel on assiste depuis l'été dernier, ne peut continuer sans avoir des conséquences profondes sur l'économie du territoire.

La croissance vertigineuse des prix de l'immobilier n'est pas uniquement le résultat de données dites objectives que l'on présente traditionnellement, à savoir une forte demande (arrivée chaque jour de 150 émigrés de Chine, demandes émanant des entreprises étrangères) et une faible offre contrainte par des données physiques (limites du territoire, capacités de construction). En fait, les autorités de la RAS disposent d'un réservoir de terrain considérable et plusieurs analyses estiment qu'à densité identique, Hong Kong pourrait doubler sa population si la quantité de terrain vendue chaque année augmentait ${ }^{2 \prime}$. La croissance des prix de l'immobilier est avant tout le résultat d'une politique délibérée poursuivie depuis 1985 par l'administration britannique avec l'assentiment de Pékin. La signature de la Déclaration conjointe en 1984 prévoyait qu'à partir de 1985, les ventes de terrain ne pourraient dépasser 50 hectares par an. En restreignant la quantité de terrains vendus, alors que les croissances économique et démographique continuaient à progresser rapidement (environ 200000 personnes par an sur le territoire, soit une croissance de 2 à $3 \%$ ), les prix de vente se sont envolés très rapidement à partir de 1987. Depuis cette date, l'immobilier est devenu un moyen d'enrichissement pour tous les acteurs de la vie économique du territoire.

Le premier bénéficiaire a été d abord le gouvernement qui, grâce à la montée des prix de vente des terrains et les différents impôts liés à l'immobilier, a continué d'assurer en moyenne près de $30 \%$ de ses revenus fiscaux sur l'immobilier et le foncier ${ }^{22}$. Les deuxièmes, et certainement les plus importants bénéficiaires, ont été les promoteurs immobiliers avec, dans leurs sillons, les grandes compagnies de construction. Une partie de la construction du parc immobilier a été laissée à partir de 1988 entre les mains des promoteurs privés ${ }^{\text {th. }}$. Les sommes très importantes que devaient réunir les promoteurs pour acquérir des terrains, vendus aux enchères. ont très rapidement créé des barrières à l'entrée en matière de financement et contribué à créer un marché oligopolistique : sept groupes (Cheung Kong dirigé par le milliardaire Li Ka-shing, Sun Hung Kai, Henderson. Sino Land. Swire. Wheelock \& Co et New World) se partagent, depuis, l'essentiel du marché. La quantité limitée d'appartements mis en vente sur le marché a permis à ces promoteurs de s'assurer des marges de profit phénoménales avec des taux de retour sur investissement qui ont pu atteindre, certaines années, près de $100 \%$. La troisième catégorie de bénéficiaires est plus difficile à cerner; mais certains ménages, disposant d'une capacité d'épargne importante, ont eux aussi bénéficié largement de cette croissance de l'immobilier. Dans un contexte d'anticipation à la hausse des prix de l'immobilier, les achats d'appartements sont devenus le placement financier le plus prisé par les ménages à Hong Kong. Une classe de rentiers, vivant sur l'immobilier, s'est rapidement développée depuis le milieu des années 80 , et bien qu'il soit difficile d'obtenir des informations pour mieux cerner cette population, les revenus des personnes privées, provenant de la location, représentaient $12,7 \%$ du PNB en 1995 , soit plus que la finance $(10,6 \%)$ ou le commerce de détail $(5 \%)^{(5)}$. Les faibles taux d'intérêts réels, (voire parfois négatifs), ont incité les ménages à investir dans l’immobilier, les loyers étant parfois plus élevés que les remboursements de crédits hypothécaires. Dans un contexte où il n'existe pas de système de retraite digne de ce nom, les ménages ont assimilé l'immobilier à un produit financier similaire aux obligations ou aux actions pour se prémunir contre le futur. Ceci à tel point que la notion d'accession à la propriété est, dans bien des cas, une motivation secondaire dans les décisions d'achat, la frontière entre spéculateur-investisseur et utilisateur final étant de plus en plus floue. Ce n'est donc pas un hasard si, dans la structure des actifs financiers à Hong kong, pour l'année 1995, l'immobilier et le foncier arrivaient de loin en tête représentant $302 \%$ du PNB, devant les actions avec une capitalisation boursière d'un montant égal à $217 \%$ du PNB et un marché obligataire quasiment inexistant d'un montant équivalent à $21 \%$ du PNB ${ }^{\prime \prime}$. Les banques ont également bénéficié du développement de l'immobilier. Ln peu plus de $40 \%$ (la limite officielle de $40 \%$ a élé franchie en 1997 pour atteindre près de $50 \%$ selon les experts) de leur activité de crédit est dominée par le crédit à l'immobilier, leur assurant ainsi une source de revenus importante. Enfin, le dernier chaînon de cette belle mécanique réside dans le marché boursier. Les marges importantes réalisées dans le secteur immobilier ont incité les investisseurs à placer des sommes importantes sur les valeurs liées à ce secteur. Cela a permis aux promoteurs immobiliers de lever, parallèlement aux crédits bancaires syndiqués, des sommes considérables sur la bourse de Hong Kong pour leurs besoins de financement. On estime de ce fait que l'immobilier domine très largement la bourse de Hong Kong avec $60 \%$ du total de la capitalisation boursière". Dans ce contexte. tous les principaux acteurs de la vie économique de Hong Kong ont 
contribué, par leurs actions à activer cette "pompe à richesse », laissant dans le logement public subventionné, représentant près de $50 \%$ de la population, ceux qui n'avaient pas les moyens d'entrer dans la ronde. $\mathrm{Ni}$ le gouvernement hritannique, voulant laisser une image de bon gestionnaire derrière lui, ni celui de Pékin, soucieux de garantir une rétrocession sans accrocs et de s'assurer l'amitié du monde des affaires, n’ont voulu s'attaquer au problème de la montée vertigineuse des prix de l'immobilier, chacun se rangeant derrière les données, dites objectives, de l'offre et de la demande.

Hong Kong est certes loin d'avoir réalisé les mêmes erreurs que les autres pays asiatiques en matière d'immobilier. Les banques ont été soumises à un contrôle plus sévère sur leur activité de crédit par les autorités monétaires ; elles disposent, également, de ratios de liquidité beaucoup plus élevés qu'au Japon avant l'éclatement de la bulle $(17,8 \%$ en moyenne à Hong Kong alors qu'au Japon ce ratio était, en 1990, seulement de $8 \%$ ), et les défauts de paiement restent, pour l'instant, à un niveau très faible, moins de $1 \%$, même s'il est possible qu'ils augmentent rapidement avec la montée du chômage ${ }^{\mid x_{1}}$. Il y a donc des risques très limités d'une crise de liquidité généralisée du système bancaire intérieur. Cependant, cette activité frénétique sur le marché de l'immobilier n’est pas sans avoir eu des effets négatifs sur l'économie du territoire et cela bien avant le début de la crise asiatique. Une étude récente estimait que, juste avant la chute des prix de l'immobilier, la moyenne des loyers pour les commerçants à Hong Kong était $41 \%$ plus élevée que les loyers pratiqués dans les quartiers les plus chers de New York ". . Certains hôtels à Hong Kong demandaient à leurs locataires détaillants, installés dans leurs murs, des prix atteignant le double de ce qui est pratiqué à Londres ou à Tokyo "'t". Les prix de l'immobilier se sont répercutés sur les prix de ventes des biens, faisant de Hong Kong un des centres commerciaux le plus cher du monde. Le coût d'un expatrié ou de la main-d'cuvre qualifiée est parmi l'un des plus élevés au monde, là encore juste derrière Tokyo : un professeur d'université coûte 250000 SUS annuellement et un cadre moyen 65000 \$US "'. Aussi, au premier retournement de conjoncture ou de baisse de la confiance sur les atouts du territoire, de tels prix ont eu très rapidement des effets négatifs sur le tourisme, le commerce de détail et les décisions d'implantation des entreprises étrangères. dans un environnement où les dévaluations ont rendu encore un peu moins chères les principales capitales asiatiques. Ce ne sont cependant pas là les seuls effets négatifs sur l'économie de Hong Kong.

Etant donné l’importance de l’immobilier dans les revenus des acteurs économiques (gouvernement. ménages, banques, actionnaires. entreprises), une correction sur les prix telle que celle que nous connaissons aujourd'hui. a des effets dévastateurs sur l'économie, créant des destructions de richesses colossales. Une étude. publiée par la banque Standard Chartered. esti- mait que la baisse des valeurs boursières et des prix de l'immobilier, depuis le début de la crise, aurait conduit à une destruction de richesse équivalente à 4300 milliards de \$HK soit un peu plus de trois fois le PNB de Hong Kong '2'. Les conséquences sur la consommation et l'activité des banques sont immédiates, comme le montre tous les indicateurs publiés depuis quelques mois. Enfin, les pratiques spéculatives qui ont accompagné le développement de l'immobilier ont créé une économie de rente avec des recyclages de ressources sur des activités autres que les activités productives, rendant Hong Kong d'autant plus dépendante de l'immobilier.

Ce constat a été d'ailleurs très vite réalisé par la nouvelle administration de la RAS. Le programme de politique générale de Tung Chee-hwa, annoncé au mois d'octobre, consistait à casser la spirale spéculative avec la construction de 85000 logements par an, dont une bonne partie serait assurée par le secteur public. L'idée de Tung Cheehwa était de crever en douceur la bulle immobilière, favoriser les revenus du travail et orienter le recyclage des ressources sur des activités productives à forte valeur ajoutée, s’inspirant clairement de la politique poursuivie à Singapour ${ }^{\prime \prime 3}$. Cependant, le programme du nouveau chef de l'exécutif n'avait pas anticipé la crise asiatique. Celleci a accéléré et amplifié, par le biais de la hausse des taux d'intérêts, les effets du dégonflement de la bulle immobilière. Le gouvernement veut désormais éviter de voir les prix s'effondrer trop rapidement ne pouvant agir sur les taux d'intérêts, en raison de la situation monétaire asiatique. Un dégonflement progressif des prix permet, en effet, de répartir les pertes du dégonflement sur plusieurs individus ou institutions, dans la mesure où le propriétaire a encore la possibilité de revendre son bien immobilier. Par ailleurs, la valeur du bien immobilier, après une faible chute des prix, a moins de risques d'être inférieure au montant du prêt hypothécaire accordé par les banques, celles-ci étant dans ce contexte moins enclines à retirer leur crédit. Par contre, dans le cas d'une chute rapide des prix, les acheteurs potentiels disparaissent (comme c'est le cas aujourd'hui) et le coût est supporté par une seule personne. La valeur du bien immobilier devient bien souvent inférieure au montant du crédit accordé par les banques; celles-ci sont incitées à retirer leur crédit, plaçant les individus dans une situation financière difficile, impliquant bien souvent une renégociation des contrats ou tout simplement des défauts de paiement.

Malgré les mesures décidées le 23 juin dernier par le gouvernement de la RAS, visant à stabiliser les prix par l’arrêt des ventes de terrains jusqu à la fin mars 1999 (fin de l'année fiscale), il y a fort à parier qu'on ne reverra pas apparaître de sitôt un mouvement de flambée des prix de l“immobilier tel qu“il s’est produit entre 1993 et 1995 , et à partir de la fin 1996 jusqu à l’été 1997. A court terme d’ahord, l'instabilité monétaire en Asie risque de se poursuivre. Les banques locales, comme les étrangères, ont déjà commencé à adopter une politique de réduction de 
leur exposition. notamment dans l'immobilier. Alors que durant. le mois de juillet 1997. elles avaient accordé un montant de 3.85 milliards de SHK de prêts liés à l'immobilier. elles n'avaient prêté. au mois d'avril dernier. que 1,32 milliard de $\$ H^{\prime 1+}$. Les taux d'intérêts devraient également rester à un niveau élevé, maintenant le coût des crédits hypothécaires à un niveau supérieur aux loyers et limitant la hausse des valeurs boursières. A plus long terme, cependant, à moins d'un revirement spectaculaire de la politique de Tung Chee-hwa, la population de Hong Kong sait désormais que les récentes mesures ne sont que temporaires et que l'objectif à long terme du nouveau gouvernement reste de diminuer les prix de l'immobilier et de limiter les pratiques spéculatives dans ce secteur. Par ailleurs, si la parité du SHK se maintient à ce niveau. une nouvelle montée des prix de l'immobilier deviendrait difficilement envisageable, dans une Asie où toutes les monnaies ont été dévaluées. Dans ce contexte. il semble bien que ce secteur ne pourra plus être le principal moteur de la croissance économique, comme cela a été le cas ces dix dernières années, ce qui pourrait entraîner de profondes conséquences sur l'économie du territoire.

\section{Les évolutions structurelles liées à la rétrocession}

Au moment de la rétrocession, bon nombre d'interrogations avaient été soulevées sur l'avenir économique du territoire. La plupart d'entre elles restent plus que jamais d'actualité dans la mesure où la crise actuelle pourrait précipiter des changements structurels liés à ce changement de statut politique de Hong Kong. Parmi ces différentes interrogations, la question de la complémentarité des deux économies reste centrale. du point de vue de la division du travail et des infrastructures et du point de vue financier.

Du point de vue de la complémentarité financière. la période de la rétrocession avait donné lieu à l'établissement de grands plans pour le futur de Hong Kong. La RAS devait notamment jouer un rôle crucial dans le financement des restructurations et des privatisations du secteur d'Etat chinois, ainsi que dans le développement de certaines grandes entreprises disposant de filiales à Hong Kong (les Red Chips). Les entreprises chinoises étaient censées continuer sur leur lancée du début des années 90 durant lesquelles elles ont connu une croissance exponentielle de leurs capacités de financement. Une analyse récente estimait que les entreprises chinoises installées à Hong Kong (les entreprises d'Etat distribuant des $H$ shares, les Red Chips cotées en bourse ou non) auraient levé sur la bourse et le système bancaire local entre 40 et 60 milliards de SUS entre le début des années 80 et l'année 1995. soit pour la même période la moitié de lendettement extérieur de la Chine ". De ce point de vue. la période post-rétrocession a mal commencé. Les entreprises chinoises cotées à la bourse de Hong Kong ont vu leurs cours s'effondrer : l'indice des entreprises chinoises a été divisé par quatre entre le mois de juillet 1997 et le mois de juin 1998. soit deux fois plus que la baisse enregistrée par l'indice général Hang Seng. Comme nous le précisions dans l"introduction. les nouvelles cotations d'entreprises d'Etat et les opérations d augmentation de capital de la part des Red Chips à la bourse de Hong Kong se font désormais au comptegouttes depuis le début de la crise asiatique.

La question qui se pose alors est de savoir si tout redeviendra comme avant une fois que les désordres monétaires en Asie se seront calmés? Les responsables de la Bourse de Hong Kong restent d'un optimisme presque déconcertant dans l'environnement actuel. en annonçant récemment leur désir de propulser l’indice Hang Seng aux alentours de $2000(0)$ points en l'espace de 5 ans (il oscille aujourd hui entre 7500 et 8500 après être monté à 16673 en août dernier) avec la cotation de quelque 150 entreprises chinoises supplémentaires (en plus des 100 cotées actuellement). Bien (yu' un retournement complet de situation soit toujours possible. un tel projet semble plutôt destiné à relever le moral des troupes que représenter un objectif réalisable à moyen terme. Et ceci pour plusieurs raisons. La crise actuelle devrait en effet laisser des traces du côté des investisseurs. D'une part, sur la population locale qui a parfois littéralement brûlé son épargne en investissant dans ces entreprises. D’autre part. sur les investisseurs institutionnels qui risquent désormais d'avoir des exigences beaucoup plus sévères en matière de transparence. Les Red Chips et $H$ shares n'en sont pas à leur première crise de confiance ${ }^{\prime k n}$. En 1990, puis en 1995. les investisseurs avaient estimé que ces entreprises étaient entachées de coûts de transaction bien trop élevés pour pouvoir y investir en toute confiance. Cependant. avant la crise asiatique. des phénomènes comme la difficulté d'obtenir des comptes consolidés. l'utilisation détournée et spéculative de fonds levés en bourse, destinés originellement à des investissements productifs, apparaissaient comme des risques importants. mais acceptables compte tenu du rythme de la croissance dans la région. Ces risques sont désormais très mal tolérés par les investisseurs depuis les récents événements en Corée du Sud, au Japon et en Asie du Sud-est. La barre sur laquelle sont placées les exigences des investisseurs (notamment institutionnels). en matière de transparence, d'appréciation de la valeur réelle des actifs situés en Chine des entreprises chinoises à Hong Kong. est désormais beaucoup plus haute depuis le début de la crise et risque de le rester dans le futur. Certaines red chips ont un endettement si élevé. comme la Shanghai Industrial Investment (ratio dettes sur capital égal à 380 \%). Guangdong Enterprises Holding (2.37\%) ou Cosco (21.3\%) que certains analystes ont qualifié leur situation financière de "bilan à la coréenne" . Pall ailleurs, on alssiste depuis le début de la crise à un renversement de situation en matière de coûts des financements externes entre Hong Kong et la Chine. Alors que. pendant des années, le coût du crédit était sensiblement moins élevé à Hong Kong. la hausse des taux d’in- 
térêts dans la RAS et la baisse des taux en Chine rendent désormais plus avantageux. pour les entreprises chinoises, de se financer directement en Chine. I à encore. la durée de l'instabilité monétaire en Asie et la question de la parité du \$HK joueront un rôle central dans cette différence des taux d'intérêts entre Hong Kong et la Chine. Enfin, il reste à préciser que les entreprises chinoises installées à Hong Kong ont été à la fois les actrices et les bénéficiaires les plus importantes de cette croissance de l'immobilier. Lors du boom de l'immobilier en 1994. elles réalisaient un peu plus de

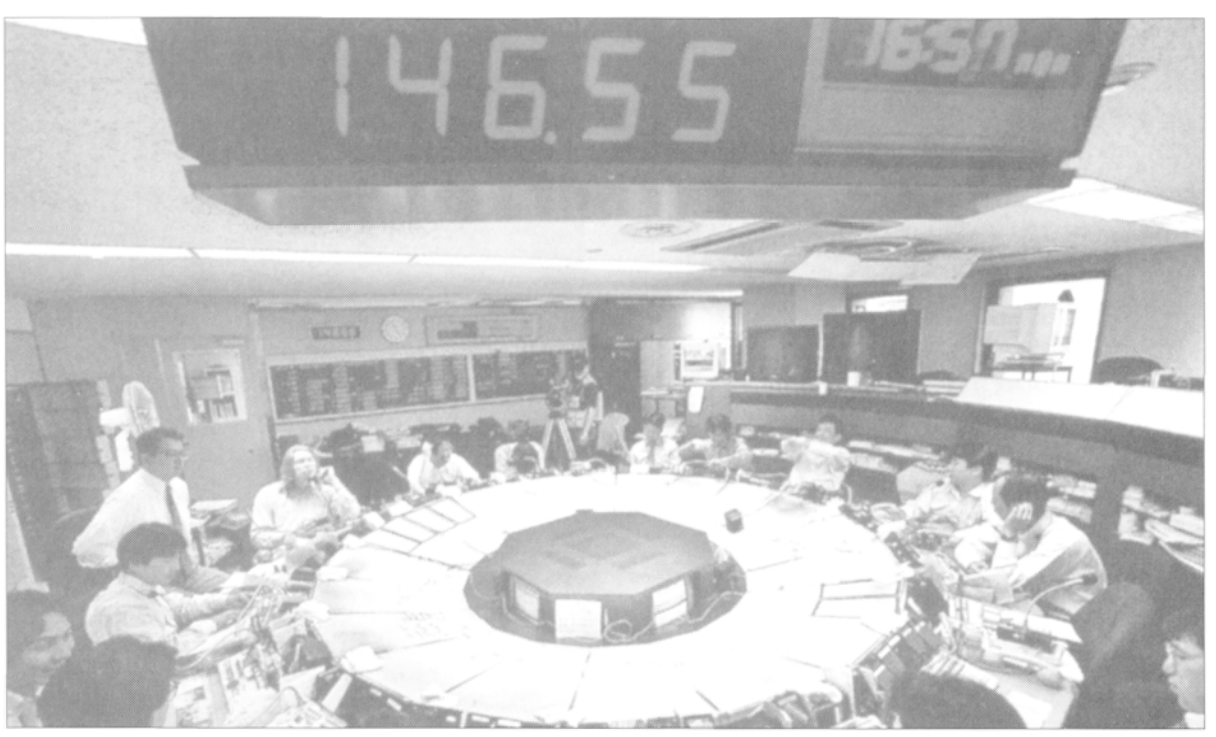

La turbulence monétaire en Asie augmente les incertitudes sur le Hong Kong dollar

$30 \%$ des transactions immobilières" ". Si. comme nous le pensons, ce secteur voit son importance décroître dans l'économie du territoire dans les années à venir, les conséquences sur la santé financière et les stratégies d'investissements des firmes chinoises installées à Hong Kong devraient se faire sentir. Ce nest ainsi pas un hasard si. durant le mois de juin dernier. l'agence de classement internationale Moody's Investors Service a mis sous revue le classement de sept sociétés-mères d'entreprises chinoises installées à Hong Kong, notamment le groupe CITIC Pékin, maison-mère de CITIC Pacific, un des fleurons des Red Chips à Hong Kong ' ${ }^{\prime 11}$.

Pour toutes les raisons invoquées ci-dessus, nous pensons que la crise asiatique est en train de précipiter l'épuisement d'un modèle de développement qui s"était mis en place progressivement entre le premier choc pétrolier en 1974 et la signature de l'accord commun entre la Chine et l'Angleterre en 1984. De plus en plus d'analyses prennent désormais la crise de 1974. plutôt que celles de 1984, 1987, 1989. et 1995. comme point de référence, pour comprendre la situation actuelle ${ }^{2 t}$. La crise de 1974 avait amorcé des ajustements structurels dans l'économie hongkongaise qui n'ont pris un visage définitif que durant la deuxième moitié des années 80). avec comme grands axes la réorientation progressive de l'activité de l’industrie vers les services. le transfert de l'industrie dans la province du Guangdong et le développement d'une nouvelle politique de l'immobilier. Une période similaire pourrait s"ouvrir actuellement par le biais dajustements et de la recherche de nouvelles niches de croissance.

\section{Atouts et incertitudes dans les politiques de sortie de crise}

Hong Kong est loin d’être dépourvue d'atouts pour sortir transformée de cette crise. Outre l'importance de ses réserves et la santé de son système bancaire. le gouvernement pourrait. à moyen et long termes. continuer par

certaines mesures à transformer les structures de fonctionnement de léconomie. La question de la politique industrielle et technologique, qui a déchainné des débats houleux sur le territoire, s"est un peu trop focalisée sur la construction du fameux parc technologique qui devrait être terminé en 2013. Or. même si on ne réinvente pas des compétences et une spécialisation territoriale en quelques années. Hong Kong semble avoir tout à gagner de politiques visant à aider les entreprises du territoire à proposer des biens et services à plus forte valeur ajoutée dans un environnement concurrentiel : formation des ressources humaines, aide aux financements pour les PME innovantes, politique de la concurrence. Toutes ces politiques pourraient permettre aux entreprises locales de remonter dans la chaîne de la valeur ajoutée et de mieux gérer une spécialisation complémentaire avec la Chine, comme le prouvent de nombreux succès d'entreprises hongkongaises dans le textile ou l'électronique':2'. La création d'un deuxième marché sur la bourse de Hong Kong pour ces PME innovantes, pourrait non seulement aider les entreprises locales, mais également attirer des firmes de la région et renforcer le rôle d'intermédiation financière de Hong Kong. Plusieurs PME taiwanaises installées en Chine auraient déjà montré un intérêt pour ce projet (22). Par ailleurs, soucieux de relancer l'activité dans la RAS. Pékin pourrait aider les investisseurs de Hong Kong en leur offrant un accès privilégié aux régions de l'intéricur. et en accélérant encore un peu plus la coopération avec la province du Guangdong. notamment sur le plan des infrastructures et de la technologie.

Il reste néanmoins beaucoup d'incertitudes sur la capacité de la RAS à sortir rapidement de la crise et à reconstruire de nouveaux piliers de croissance durables. A la différence de ce qui s'est passé après la crise de 1974 où le développement rapide des exportations avait facilité les ajustements structurels, les initiatives du gouvernement et du secteur privé se heurteront. durant les prochaines années. à un environnement plus difficile comp- 


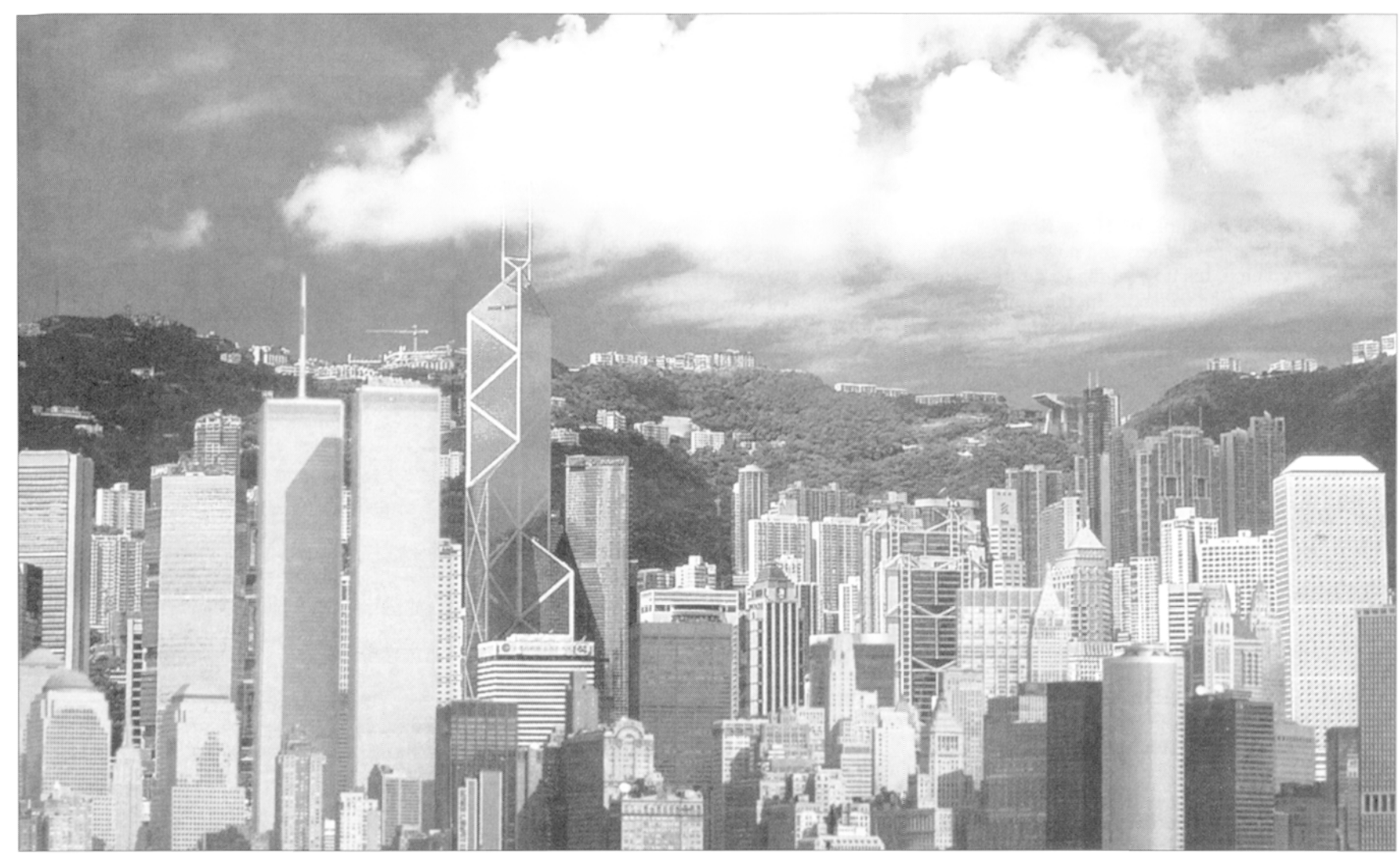

Le dégonflement de la bulle immobilière a fait déjà beaucoup de victimes à Hong Kong

te tenu du contexte économique régional déprimé. Parallèlement, l'économie de Hong Kong devra certainement continuer à court et moyen termes à subir les conséquences de l'ajustement drastique des prix de l'immobilier qui a commencé depuis l'automne 1997. A part quelques voix dissonantes émanant des promoteurs immobiliers, la plupart des analyses s'accordent en effet pour reconnaître, dans la lignée du programme du chef de l'exécutif annoncé en octobre 1997, qu'il faut continuer à dégonfler la bulle immobilière. Seule une telle politique permettrait de rendre Hong Kong plus compétitive, de limiter les comportements spéculatifs tout en réorientant les ressources sur des investissements productifs. Mais cela permettrait surtout de relancer la consommation en réduisant ce formidable impôt déguisé sous la forme des prix d'achat exorbitants des logements (et par répercussion des biens et des services) qui pèse sur le dos des ménages.

Cependant, une fois ce constat réalisé, il s'avère que beaucoup de Hongkongais doutent désormais de la capacité du gouvernement à imposer les ajustements nécessaires à certaines catégories qui ont largement profité de la politique des prix élevés de l'immobilier, à savoir les promoteurs immobiliers et les propriétaires rentiers. On comprend, pour les raisons indiquées plus haut, pourquoi le gouvernement cherche à éviter un effondrement trop rapide des prix de l'immobilier. Cependant, les récentes décisions de bloquer les ventes de terrain ont été largement interprétées comme un cadeau fait aux promoteurs immobiliers, qui étaient sortis à plusieurs reprises de leur neutralité politique pour dénoncer la politique du gouvernement de la RAS en matière de logement ${ }^{2 *}$, alors qu au même moment, les différentes catégories de propriétaires continuaient de refuser un ajustement des prix des loyers demandés par les commerçants locataires. Les coalitions de commerçants locataires se sont multipliées, depuis ces derniers mois, pour demander à la fois au gouvernement et aux promoteurs des réductions de loyers. Certains centres commerciaux ont des taux de vacances atteignant 40 à $50 \%$ avec le départ de plusieurs détaillants qui ne peuvent plus payer leurs loyers avec la chute des ventes. Leurs demandes cependant sont loin d'avoir été exaucées, les propriétaires offrant des réductions de 10 à $15 \%$ dans le meilleur des cas (certains, ayant signé leur contrat de location en 1996, se voient appliquer des augmentations. les propriétaires estimant que les prix du marché sont supérieurs aujourd hui par rapport à 1996), alors que les associations estiment qu il faudrait des réductions d une fourchette allant de 30 à $60 \%$, selon les cas, pour pouvoir survivre dans un environnement économique aussi difficile'ts. Il faudra également faire accepter aux banques l'idée de crédits à l’immobilier à taux fixe qui n'existent toujours pas. De la même manière. alors que Hong Kong est un des ports qui pratique les prix les plus élevés du monde, les charges de transbordement et d'utilisation des infrastructures ont été augmentées de $20 \%$ pour le commerce à destination de l'Asie. et de $3.8 \%$ vers l'Amérique du Nord, provoquant la colère de l'association des exportateurs de Hong Kong ".". 
Sur le plan fiscal, beaucoup de questions ont été posées sur la politique du gouvernement. D'une part sur les choix opérés par celui-ci pour répondre à l'éventualité d'une diminution structurelle des recettes fiscales liées à l'immobilier qui entrânerait un déficit fiscal structurel. Pour preuve, la décision récente de geler les ventes de terrain s'est traduite automatiquement par l'annonce d'un possible déficit fiscal pour l'année 1998, alors que dans les prévisions budgétaires, le secrétaire aux Finances Donald Tsang avait prévu, au mois de février dernier et de manière surprenante, un surplus fiscal de 77 milliards de $\$ H^{\circ}{ }^{20}$. Le système du currency. board empêche le gouvernement de pratiquer une politique de création monétaire déconnectée des entrées de devises (pour chaque \$US détenu à Hong Kong, les trois banques émettrices de monnaie ont le droit de créer 7.7 \$HK). Par ailleurs, la loi fondamentale qui sert de constitution à Hong Kong interdit un déficit budgétaire structurel. Les autorités semblent, pour l'instant, jouer la politique de l'autruche et affirment que s'il y a déficit, il sera passager. Or, si la crise se maintenait sur une période relativement longue, un arbitrage devra intervenir entre une modification de la loi fondamentale ou la hausse des impôts, remettant en cause le caractère de paradis fiscal de Hong Kong. D'autre part, sur l'utilisation des réserves colossales d'un montant de 446 milliards de \$HK. Les partis politiques démocrates, qui sont revenus en force lors des élections du 24 mai dernier de la nouvelle assemblée législative, pensent qu'il est désormais temps que le gouvernement tienne ses promesses, à savoir que les réserves soient utilisées comme l'avait déclaré le secrétaire aux Finances en 1997 «lorsque le mauvais temps s'abattra sur Hong Kong ". Or, celui-ci vient une nouvelle fois de déclarer que pour des raisons de prudence fiscale, Hong Kong ne pouvait se servir de ses réserves pour atténuer les conséquences de la crise $^{127}$.

Ces questions illustrent asse $z$ bien un ensemble de problèmes liés à l'exercice de la démocratie dans la résolution des difficultés économiques et sociales issues de la crise actuelle. Plus la crise durera et plus l'absence de démocratie et de transparence qui caractérise les institutions politiques de la RAS sera critiquée par une population qui a déjà montré à plusieurs reprises, lors des élections, ses aspirations démocratiques. On peut, en effet, souhaiter que cette crise soit un moment privilégié pour repenser. de manière un peu plus démocratique que par le passé, un certain nombre de questions économiques fondamentales, comme la fiscalité, la couverture sociale ou l'immobilier. Le gouvernement de la RAS dispose encore de bonnes marges de manoxuvre tels le montant des réserves, la qualité des infrastructures, la mentalité d'émigrants qui continue à prévaloir dans les classes les plus défavorisées toujours déterminées à réussir avec peu de demandes sociales malgré une inégalité qui reste parmi les plus élevées au monde ${ }^{2 x}$. Et enfin. des effets d'agglomération des activités économiques qu'on ne supprime pas facilement et qui continuent à jouer en faveur de Hong Kong. Il faudra néanmoins que le gouvernement réagisse vite, car le temps presse et les défis s'accumulent. Il devra surtout ćvitcr de prôner comme unique politique, la réversibilité des évolutions économiques par le seul jeu de la flexibilité des marchés, réversibilité qui n’existe que dans les textes d'école.

1. Toyojiro Maruya. "Tasks for Hong Kong"s Fconomy in the New Era. Shift to a Service Oriented Economy and Introduction of a Comprehensive Competition Policy ", in Siu-Lun Wong et Toyojiro Maruya. Hong Kong Econom! and Society : Challenges in the New Era. Tokyo. Institute of Developing Economies. 1998.

2. Asian Wall Street Journal. 3 juin 1998

3. Mariko Watanabe. "Borrowed Land: How does the "rug" under the Hong Kong economy work ?" in IDE. Hong Kong 1997 : Society in Transition. Tokyo. Institute of Developing Economies. 1996.

4. Voir l'article de Lionnel Bessard dans ce numéro.

5. Toyojiro Maruya. op. cit.. p. 2.

6. Mariko Watanabe. op. cit. p. 24.

7. Marc Faber Limited. miméo, non publié.

8. Asian Wall Street Jotumal. 12 mars 1998.

9. Healey \& Baker cité dans South China Moming Post. 5 juin 1998.

10. South China Morning Post. 5 juin 1998.

11. Yi-zheng Lian "An Economic Roundup of Post-Handover Hong Kong " in Jeffrey Fischer \& al. (eds). Hong Kong : The Challenges of Change an Asia Society Publication. Asian Update. Mai 1998 : voir également le classement public dans The Economist. p. 122. 20-26 juin.

12. Citée dans Financial Times, p. 14. 29 juin 1998.

13. Xin Bao, 25 juin 1998.

14. Asian Wall Street Jolumal. 3 juin 1998.

15. Charles De Trenck. Simon Cartledge. Anil Daswani. Christian A. Kat\% et David Sakmar. Red Chips and the Globalisation of China's Enterprises, Hong Kong. Asia 2000. 1998. p. 47.

16. Jean-François Huchet. « Les entreprises chinoises à Hong Kong : des partenaires ambigus dans l'avenir du Territoire". in Perspectives Chinoises, $n^{\circ} 41$, maijuin 1997. pp. 54-66.

17. Asian Wall Street Journal. 21 avril 1998.

18. Charles De Trenck \& al.. op. cit.. p. 57.

19. Xin Bao. 8 juin 1998.

20. Voir par exemple Dong Tao, « Domestic Problems point to prolonged recession ". in South Chima Moming Post. 6 juin 1998.

21. Voir pour le textile in Far Esteram Ecomomic Revien. 21 mai 1998. pp. $52-55$.

22. Asian Wall Street Jomrnal. 8 juin 1998.

23. Ming Pao. 22 mai 1998.

24. South China Morning: Post. 5 juin 1998.

25. South China Morning Post. 3 juin 1998.

26. Asian Wall Street Journal. 19 février 1998.

27. Ming Pao. 26 juin 1998.

28. Le coefficient de Gini, yui mesure la dispersion dans la distribution des revenus était en 1996 de 0.518 . un des plus élevés parmi les pays développés (0.35 pour les pays ayant une redistribution relativement Égalitaire), en projection constante depuis le début des années 80). cité in Toyojiro Maruya op. cit.. p. 9. 